\title{
Floquet Majorana fermions and parafermions in driven Rashba nanowires
}

\author{
Manisha Thakurathi, Daniel Loss, and Jelena Klinovaja \\ Department of Physics, University of Basel, Klingelbergstrasse 82, CH-4056 Basel, Switzerland
}

(Received 28 August 2016; published 6 April 2017)

\begin{abstract}
We study a periodically driven nanowire with Rashba-like conduction and valence bands in the presence of a magnetic field. We identify topological regimes in which the noninteracting system hosts zero-energy bound states. We further investigate the effect of strong electron-electron interactions that give rise to parafermion zero energy modes hosted at the nanowire ends. The first setup we consider allows for topological phases by applying only static magnetic fields without the need of superconductivity. The second setup involves both superconductivity and time-dependent magnetic fields and supports topological phases without fine tuning of the chemical potential. Promising candidate materials are graphene nanoribbons due to their intrinsic particle-hole symmetry.
\end{abstract}

DOI: 10.1103/PhysRevB.95.155407

\section{INTRODUCTION}

Topological phases in condensed matter systems have been at the center of attention over the past decade. So far most of the studies on topological phases such as topological insulators [1-13], Majorana fermions [14-36], and parafermions [3744] were focused on static systems. However, the dearth of naturally occurring topological materials is stimulating new proposals to engineer systems with topological phases.

External driving gives us a powerful tool to turn initially nontopological materials into topological ones [45]. This is a most promising approach for both condensed matter and cold atom fields. Recently, there have been several studies in which systems driven out of equilibrium give rise to a topological Floquet spectrum [45-65]. The existence of exotic edge modes have been demonstrated by direct observation in photonic crystals [46,47]. The Floquet states have remarkably richer structure than its static counterparts. There have been proposals on various novel phases of Floquet systems such as Floquet topological insulators [45,48-50], Floquet topological superfluids [51], and Floquet Weyl semimetals [48,52]. In this work, we explore one of such phases, namely, Floquet fractional topological insulators which exhibit fractional excitations. This phase requires the presence of strong electron-electron interactions $[42,43,48]$, which is an interesting subject on its own in driven systems $[66,67]$.

In the first setup, we consider a Rashba nanowire (see Fig. 1) driven by an oscillating electric field $[\mathcal{E}(t)]$ with frequency matching the energy difference between the conduction and valence bands. We note that our results are applicable to any single-channel system such as semiconducting nanowires, graphene nanoribbons, and nanotubes [68-83]. We show that the topological zero energy bound states localized at the nanowire ends can be realized by the mere presence of a uniform static magnetic field without any need of superconductivity. This proposal is attractive experimentally as it avoids the detrimental combination of magnetic fields and superconductivity. In the second setup, a one-band Rashba nanowire with proximity-induced superconductivity is subjected to a time-dependent magnetic field. This setup has an important advantage over those with time-independent magnetic fields $[15,16]$ in that the chemical potential does not need to be tuned close to the spin-orbit energy. For both setups, we find topological bound states also in the fractional charge regime. These setups not only provide a proof-of-principle for fractional topological effects in Floquet systems but also show great promise to be experimentally implemented in realistic systems such as graphene nanoribbons.

\section{FLOQUET RASHBA NANOWIRE IN APPLIED MAGNETIC FIELD}

We consider a one-dimensional Rashba nanowire (see Fig. 1) aligned along the $x$ direction characterized by the spinorbit interaction (SOI) vector $\boldsymbol{\alpha}$, which points perpendicular to the nanowire axis in the $z$ direction. The corresponding Hamiltonian is given by

$$
H_{0}=\sum_{\eta \sigma} \eta \Psi_{\eta \sigma}^{\dagger}\left(-\frac{\hbar^{2} \partial_{x}^{2}}{2 m_{0}}+\alpha \sigma \partial_{x}+\delta_{1 \eta} \Delta_{g}\right) \Psi_{\eta \sigma} .
$$

Here, $m_{0}$ is the effective electron mass. The index $\eta=1(\eta=$ $\overline{1})$ corresponds to the conduction (valance) band and $\sigma=1$ $(\sigma=\overline{1})$ to spin up (down) states. The fermion operator $\Psi_{\eta \sigma}(x)$ annihilates at position $x$ an electron from the $\eta$ band with spin $\sigma$. In the valence band, we initially tune the chemical potential $\mu$ close to the SOI energy $E_{s o}=\hbar^{2} k_{s o}^{2} / 2 m_{0}$, where $k_{s o}=m_{0} \alpha / \hbar^{2}$ is the SOI wave vector. The gap between valence and conduction bands is $\Delta_{g}-2 E_{s o}$, as shown in Fig. 2(a). A static and uniform magnetic field $\mathbf{B}$ is applied perpendicular to the SOI vector (say, along the $x$ direction) and results in the Zeeman term

$$
H_{Z}=\Delta_{Z} \sum_{\eta \sigma \sigma^{\prime}} \Psi_{\eta \sigma}^{\dagger}\left(\sigma_{x}\right)_{\sigma \sigma^{\prime}} \Psi_{\eta \sigma^{\prime}}
$$

where $\Delta_{Z}=g \mu_{B} B$ is the Zeeman energy with $g$ being the $g$ factor and $\mu_{B}$ the Bohr magneton.

Instead of making use of the standard scheme based on superconductivity [14-16], we propose to drive the system across the bulk gap by an oscillating electric field with frequency $\omega$. When $\hbar \omega=\Delta_{g}$, a dynamical gap emerges (playing the role of a superconducting gap).

We work in the Floquet representation [64,84]. To map a time-dependent problem into a stationary one, we replace 


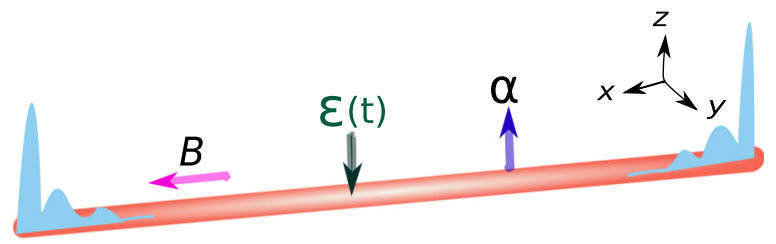

FIG. 1. One-dimensional Rashba nanowire (orange cylinder) with the SOI vector $\boldsymbol{\alpha}$ pointing in the $z$ direction is aligned along the $x$ direction. The magnetic field $\mathbf{B}$ is chosen in the $x$ direction. A driving electric ac field $\mathcal{E}(t)$ of resonant frequency $\omega$, resulting in the coupling $t_{F}$ between bands, is applied in the transverse $z$ direction. In the topological regime $\Delta_{Z}>t_{F}>0$, the system hosts zero energy bound states (blue curves) at each wire end.

the initial time-dependent periodic Hamiltonian $H(t)=$ $H(t+T)$ by the Floquet Hamiltonian $H_{F}=H(t)-i \hbar \partial_{t}$. The eigenstates of $H_{F}$ are given by the direct product of the instantaneous eigenstates $(|v\rangle \equiv|k\rangle \otimes|\eta\rangle \otimes|\sigma\rangle)$ and the set of periodic functions $e^{i n \omega t}$, where the integer $n$ defines the $n$th Floquet replica. The matrix elements then become $\left\langle v_{1} n_{1}\left|H_{F}\right| v_{2}, n_{2}\right\rangle=\left\langle v_{1}|H| v_{2}\right\rangle+n_{1} \hbar \omega \delta_{n_{1} n_{2}} \delta_{v_{1} \nu_{2}}$. We consider only the direct resonances between $n=0$ and $n=1$ involving single photon absorption/emission processes, and we work in first order approximation in the driving amplitude. The Floquet term, which couples conduction and valence bands, is given by $H_{d}=t_{F} \sum_{\eta \sigma} \Psi_{\eta \sigma}^{\dagger} \Psi_{\bar{\eta} \sigma}$, with the Floquet coupling amplitude $t_{F}=e \mathcal{E} d_{c v} / 2$ being proportional to the interband dipole term between conduction and valence band $\left(d_{c v}\right)$ and to the amplitude of the applied electric field $\mathcal{E}$ [48]. Thus, in the basis $\left(\Psi_{11}, \Psi_{1 \overline{1}}, \Psi_{\overline{1} 1}, \Psi_{\overline{1} \overline{1}}\right)$, the Floquet matrix assumes the
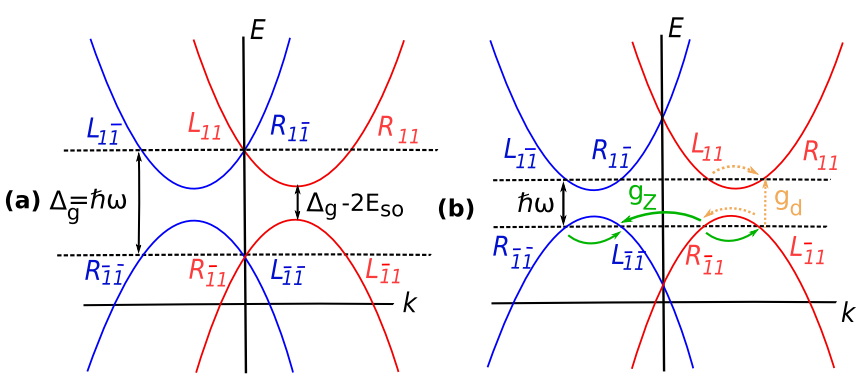

FIG. 2. The spectrum of the nanowire with band gap $\Delta_{g}-2 E_{s o}$ separating valence $(\eta=\overline{1})$ and conduction $(\eta=1)$ bands. The index $\sigma=1(\sigma=\overline{1})$ refers to the spin up (spin down) band shown in red (blue) color. The right- $\left(R_{\eta \sigma}\right)$ and left-mover $\left(L_{\eta \sigma}\right)$ fields are introduced close to the Fermi level. (a) The chemical potential $\mu$ is tuned to the SOI energy $E_{s o}$ and the driving frequency $\hbar \omega=\Delta_{g}$ results in resonant scattering between the two bands. If the Zeeman energy $\Delta_{Z}$ exceeds the Floquet amplitude $t_{F}$, the system hosts zero energy modes. (b) To obtain parafermions, we tune $\mu$ to $E_{s o} / 9$ and readjust $\omega$ to $\hbar \omega=\Delta_{g}-16 E_{s o} / 9$. The leading term in the magnetic field $H_{Z}^{e e}$ (green arrows) involves two backscattering events and opens a partial gap in the spectrum only in the presence of strong electron-electron interactions. The driving term $H_{d}^{e e}$ (yellow arrows) commutes with $H_{Z}^{e e}$ and can be ordered simultaneously in the RG sense, leading to a fully gapped spectrum.

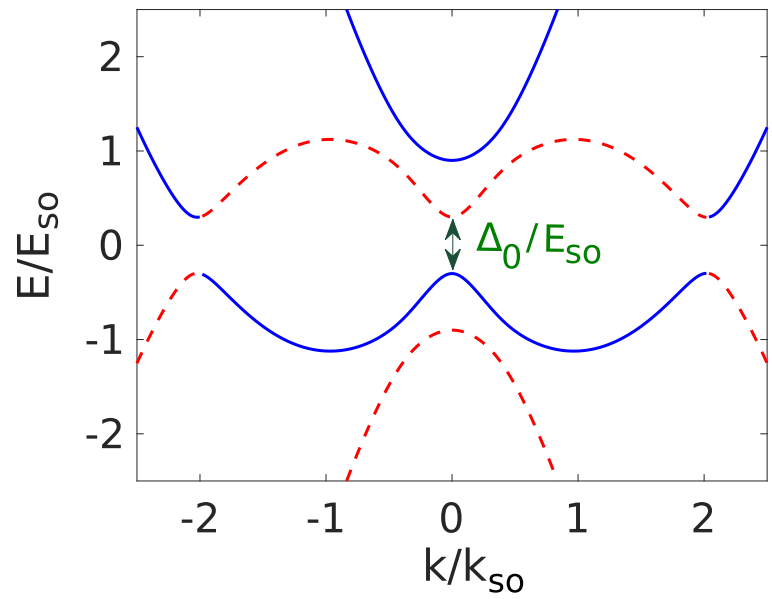

FIG. 3. Floquet spectrum [see Eq. (4)] of Rashba nanowire driven by an electric field for $\Delta_{Z} / E_{s o}=0.6$ and $t_{F} / E_{s o}=0.3$. The topological gap $\Delta_{0}=2\left|\Delta_{Z}-t_{F}\right|$ defined at $k=0$ closes for $\Delta_{Z}=t_{F}$ signaling the topological phase transition. If $t_{F}>\Delta_{Z}$, there is one zero energy bound state localized at each end of the nanowire.

form

$\mathcal{H}_{F}=\left(\begin{array}{cccc}E_{k}+\alpha k & \Delta_{Z} & t_{F} & 0 \\ \Delta_{Z} & E_{k}-\alpha k & 0 & t_{F} \\ t_{F} & 0 & -E_{k}-\alpha k & \Delta_{Z} \\ 0 & t_{F} & \Delta_{Z} & -E_{k}+\alpha k\end{array}\right)$,

where $E_{k}=\hbar^{2} k^{2} / 2 m_{0}$. We note that $\Delta_{g}$ in the upper two diagonal elements is canceled out by $\hbar \omega$. The spectrum of $\mathcal{H}_{F}$ (see Fig. 3) consists of four branches,

$$
\begin{aligned}
E_{F \pm}^{2}= & \left(\frac{\hbar^{2} k^{2}}{2 m_{0}}\right)^{2}+(\alpha k)^{2}+\Delta_{Z}^{2}+t_{F}^{2} \\
& \pm 2 \sqrt{\Delta_{Z}^{2} t_{F}^{2}+\left(\frac{\hbar^{2} k^{2}}{2 m_{0}}\right)^{2}\left[(\alpha k)^{2}+\Delta_{Z}^{2}\right]} .
\end{aligned}
$$

The gap $\Delta_{0}=2\left|\Delta_{Z}-t_{F}\right|$ at $k=0$ is zero only for $\Delta_{Z}=t_{F}$. At all other values of wave vector $k$, the gap in the Floquet spectrum is always finite. The closing of the gap $\Delta_{0}$ indicates the topological phase transition point, where for $\Delta_{Z}<t_{F}$ the phase is trivial $(v=0)$ while for $\Delta_{Z}>t_{F}$ it is topological $(v=1)$, with $v$ being the corresponding topological invariant characterizing the bulk inversion [85].

Next, we demonstrate that the system hosts zero-energy modes localized at the wire ends. For simplification, we work in the regime of strong SOI and linearize the Hamiltonian $\mathcal{H}_{F}$ [see Eq. (3)] at the Fermi surface $[86,87]$ by representing operators in terms of slowly-varying left $\left(L_{\eta \sigma}\right)$ and right mover fields $\left(R_{\eta \sigma}\right)$ defined around the Fermi points $k_{F}= \pm 2 k_{s o}$ and $k_{F}=0$ (see Fig. 2) as

$$
\Psi_{\eta \sigma}=R_{\eta \sigma} e^{i \sigma k_{s o}(1+\eta \sigma)}+L_{\eta \sigma} e^{i \sigma k_{s o}(1-\eta \sigma)} .
$$

The effective Hamiltonian density $\mathcal{H}$ is written in terms of Pauli matrices in the basis 


$$
\begin{aligned}
& \left(R_{11}, L_{11}, R_{1 \overline{1}}, L_{1 \overline{1}}, R_{\overline{1} 1}, L_{\overline{1} 1}, R_{\overline{1} \overline{1}}, L_{\overline{1} \overline{1}}\right) \text { as } \\
& \mathcal{H}=\hbar v_{F} \hat{k} \tau_{3}+t_{F} \eta_{1} \tau_{1}+\Delta_{Z}\left(\tau_{1} \sigma_{1}+\eta_{3} \tau_{2} \sigma_{2}\right) / 2,
\end{aligned}
$$

where $v_{F}$ is the Fermi velocity and $\hat{k}$ the momentum operator with eigenvalue $k$. We note that the system is assumed to be in the weak driving regime with $t_{F} \ll \Delta_{g}$. The Pauli matrices $\eta_{i}\left(\sigma_{i}\right)$ act in upper-lower (spin) spaces (subspaces) and $\tau_{i}$ act in right-left mover subspace. We note that $\mathcal{H}$ belongs to the topological class DIII [85] with the time-reversal (particle-hole) symmetry operator defined as $U_{T}=\eta_{2} \tau_{1} \sigma_{3}$ $\left(U_{C}=\sigma_{2} \eta_{2}\right)$. Being of type $\mathbb{Z}_{2}$ in $1 \mathrm{D}$, the system hosts single zero-energy modes in the middle of the gap in the topological phase unless symmetries are broken or even if, similarly to the crystalline topological insulators [88-90], the symmetries are not broken on average.

The corresponding Floquet spectrum is given by $E_{1, \pm}=$ $\pm \sqrt{\left(\hbar v_{F} k\right)^{2}+t_{F}^{2}}$ and $E_{2 \pm}^{2}=\sqrt{\left(\hbar v_{F} k\right)^{2}+\left(t_{F} \pm \Delta_{Z}\right)^{2}}$, where $E_{1, \pm}$ is twofold degenerate. If $\Delta_{Z}>t_{F}>0$, the system is in the topological phase (as found above) and hosts one zero-energy bound state with the localization lengths $\xi_{t}=\hbar v_{F} / t_{F}$ and $\xi_{-}=\hbar v_{F} /\left(\Delta_{Z}-t_{F}\right)$ at each wire end, see Appendix A for details.

We note that if there is disorder in the nanowire which locally breaks the particle-hole symmetry, for example, due to random disorder in the on-site energy $\mu(x)=\mu_{0}+\delta \mu(x)$, the fractional fermion bound states could be shifted away from zero energy, as was already studied both analytically and numerically in Ref. [91]. For example, if there is a single local impurity at $x_{0}$, this perturbation acts like a level detuning and shifts the energy level proportionally to the strength of impurity $\delta \mu\left(x_{0}\right)$ and to the occupation probability at the site of fluctuation $\left(\left|f\left(x_{0}\right)\right|^{2} \ll 1\right)$. If there are many impurities in the nanowire, we assume a fluctuation distribution such that $\int d x \delta \mu(x)=0$ but $\int d x[\delta \mu(x)]^{2} \neq 0$ and characterized by the disorder correlation length $\xi_{d}$. The ratio of the localization length of the bound state $\xi_{t,-}$ and $\xi_{d}$ plays now an important role. In first order perturbation theory (assuming weak disorder) the energy shift is given by $\delta E=\int d x|f(x)|^{2} \delta \mu(x)$ where the integration runs over the entire wire. If $\xi_{d} \ll \xi_{t}$, disorder effects are averaged out in the integration such that $\delta E \approx 0$ and the bound state stays at zero energy. In the opposite regime, $\xi_{d} \gtrsim \xi_{t}$, one deals with a quasiconstant local potential, which can give rise to a bias between left and right ends of the nanowire. In this case the bound state energy will be shifted correspondingly, resulting in the right and left bound state being at different energies, and, thus, the degeneracy will be lifted. However, we note that the nanowires should be stable against such fluctuations, as local differences in $\mu$ would lead to a charge redistribution, restoring a uniform chemical potential in the nanowire. We further note that in such situations the local shift of the chemical potential can be compensated externally by tuning local gates.

Finally, it is worth mentioning that a slight asymmetry between electron and hole bands, such as the one arising in graphene if, for instance, the next nearest neighbor hopping is taken into account [92], will not affect our result as long as the energy difference due to this asymmetry stays smaller than the gaps opened at the Fermi level. In this case, our effective Hamiltonian is the same.

\section{FLOQUET PARAFERMIONS}

The topological phases can also be realized in an interacting system, giving rise to fractional Floquet modes. For example, if the chemical potential is moved down to $\mu_{1 / 3}=E_{s o} / 9$, such that the Fermi wave vectors are given by $\pm k_{s o}(1 \pm 1 / 3)$, the nanowire hosts parafermions as we show next. We note that we work in the high frequency limit meaning that the driving frequency $\omega$ is larger than the Fermi energy [45], and electronelectron interactions can be treated with standard bosonization techniques.

Similarly to the noninteracting model, we assume that the Zeeman field is the dominant term and drives the system into the topological phase. The term which conserves both spin and momentum and is lowest order in $\Delta_{Z}$ is given by

$$
\begin{aligned}
H_{Z}^{e e}= & g_{Z}\left[\left(R_{1 \overline{1}}^{\dagger} L_{11}\right)\left(R_{1 \overline{1}}^{\dagger} L_{1 \overline{1}}\right)\left(R_{11}^{\dagger} L_{11}\right)\right. \\
& \left.+\left(R_{\overline{1} 1}^{\dagger} L_{\overline{1} \overline{1}}\right)\left(R_{\overline{1} 1}^{\dagger} L_{\overline{1} 1}\right)\left(R_{\overline{1} \overline{1}}^{\dagger} L_{\overline{1} \overline{1}}\right)+\text { H.c. }\right],
\end{aligned}
$$

where $g_{Z} \propto \Delta_{Z} g_{B}^{2}$ and $g_{B}$ is the electron-electron backscattering amplitude. This process involves the backscattering of two electrons [42,43]. Again, the frequency of the driving term matches the energy difference between the conduction and valence bands, see Fig. 2(b). For weak driving, it is sufficient to include electron-electron interactions inside each of the two bands. The term, which commutes with $H_{Z}^{e e}$ and satisfies the momentum and energy conservation laws resulting in the dynamic gap, is written as

$$
\begin{aligned}
H_{t}^{e e}= & g_{d}\left[\left(R_{11}^{\dagger} L_{\overline{1} 1}\right)\left(R_{11}^{\dagger} L_{11}\right)\left(R_{\overline{1} 1}^{\dagger} L_{\overline{1} 1}\right)\right. \\
& \left.+\left(R_{\overline{1} \overline{1}}^{\dagger} L_{1 \overline{1}}\right)\left(R_{\overline{1} \overline{1}}^{\dagger} L_{\overline{1} \overline{1}}\right)\left(R_{1 \overline{1}}^{\dagger} L_{1 \overline{1}}\right)+\text { H.c. }\right]
\end{aligned}
$$

where $g_{d} \propto t_{F} g_{B}^{2}$. We assume that Eqs. (7) and (8) are relevant in the sense of the renormalization group (RG) theory either due to their scaling dimensions or due to their initial amplitude being of order one [41-44].

We first define standard bosonic fields $\phi_{r \eta \sigma}$ as $R_{\eta \sigma}=e^{i \phi_{1 \eta \sigma}}$ and $L_{\eta \sigma}=e^{i \phi_{\bar{I} \eta \sigma}}$ with the only nonvanishing commutation relation given by $\left[\phi_{r \eta \sigma}(x), \phi_{r^{\prime} \eta^{\prime} \sigma^{\prime}}\left(x^{\prime}\right)\right]=$ $i \pi r \delta_{r r^{\prime}} \delta_{\eta \eta^{\prime}} \delta_{\sigma \sigma^{\prime}} \operatorname{sgn}\left(x-x^{\prime}\right)$. However, the problem is described better in terms of new bosonic fields $\tilde{\phi}_{r \eta \sigma}=\left(2 \phi_{r \eta \sigma}-\phi_{\bar{r} \eta \sigma}\right) / 3$ with $\left[\tilde{\phi}_{r \eta \sigma}(x), \tilde{\phi}_{r^{\prime} \eta^{\prime} \sigma^{\prime}}\left(x^{\prime}\right)\right]=i r(\pi / 3) \delta_{r r^{\prime}} \delta_{\eta \eta^{\prime}} \delta_{\sigma \sigma^{\prime}} \operatorname{sgn}\left(x-x^{\prime}\right)$.

The nonquadratic Hamiltonians $H_{Z}^{e e}$ and $H_{t}^{e e}$ [see Eqs. (7) and (8)] can be expressed in bosonized form as

$$
\begin{aligned}
& H_{Z}^{e e}=2 g_{Z} \sum_{\eta} \cos \left[3\left(\tilde{\phi}_{\eta \eta \overline{1}}-\tilde{\phi}_{\bar{\eta} \eta 1}\right)\right], \\
& H_{t}^{e e}=2 g_{d} \sum_{\eta} \cos \left[3\left(\tilde{\phi}_{1 \eta \eta}-\tilde{\phi}_{\overline{1} \bar{\eta} \eta}\right)\right] .
\end{aligned}
$$

Next, aiming to find bound states, one needs to impose vanishing boundary conditions which is best done by the following unfolding procedure $[42,43]$. We enlarge the nanowire from $[0, L]$ to $[-L, L]$ and define new fields such that the vanishing boundary conditions are satisfied automatically,

$$
\chi_{\eta \sigma}(x)=\left\{\begin{array}{ll}
\tilde{\phi}_{(\bar{\eta}) \eta \sigma}(x), & x>0 \\
\tilde{\phi}_{(\eta \sigma) \eta \sigma}(-x)+\pi, & x<0
\end{array} .\right.
$$




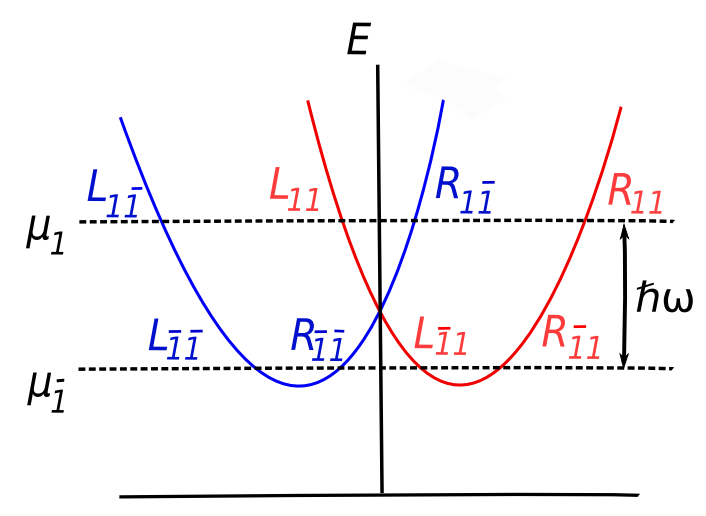

k

FIG. 4. The spectrum of the Rashba nanowire used in the second model. The index $\eta=1(\eta=\overline{1})$ is for upper and lower band, $\sigma=$ $1(\sigma=\overline{1})$ for spin up (red) [down (blue)]. The chemical potentials $\mu_{1, \overline{1}}$ and the driving frequency $\omega$ of field $B(t)$ are chosen in such a way that the smallest Fermi wave vectors of two effective subbands coincide.

Next, we define the conjugated fields $\phi_{1}=\frac{3}{2} \sum_{\eta \sigma} \chi_{\eta \sigma}, \theta_{1}=$ $\frac{3}{2} \sum_{\eta \sigma} \eta \sigma \chi_{\eta \sigma}, \phi_{2}=\frac{1}{2} \sum_{\eta \sigma} \eta \chi_{\eta \sigma}$, and $\theta_{2}=\frac{1}{2} \sum_{\eta \sigma} \sigma \chi_{\eta \sigma}$. The Hamiltonians take the form

$$
\begin{aligned}
& H_{Z}^{e e}=4 g_{Z} \cos \left(\theta_{1}\right) \cos \left(3 \theta_{2}\right), x>0, \\
& H_{t}^{e e}=4 g_{d} \cos \left(\theta_{1}\right) \cos \left(3 \phi_{2}\right), x<0 .
\end{aligned}
$$

To minimize the energy for strong coupling, the fields get pinned. Here, $\theta_{1}$ is uniform, $\theta_{1}=\pi \hat{M}$, where $\hat{M}$ is an integer-valued operator. The second field cannot be pinned uniformly and changes from $\theta_{2}=\pi(1+\hat{M}+2 \hat{l}) / 3$ for $x>0$ to $\phi_{2}=\pi(1+\hat{M}+2 \hat{n}) / 3$ for $x<0$, where $\hat{l}$ and $\hat{n}$ are integer-valued noncommuting operators with $[\hat{n}, \hat{l}]=3 i / 4 \pi$. The domain wall at $x=0$ hosts a zero-energy parafermion state $[42,43]$ defined by the operator

$$
\alpha_{ \pm}=e^{i 4 \pi(\hat{n} \pm \hat{l}) / 3}, \alpha_{ \pm}^{3}=1 .
$$

We note here that coming back to the time-independent laboratory frame, the energy of the bound states will stay at zero but the many-body wave functions will be periodically changing in time.

\section{FLOQUET RASHBA NANOWIRE PROXIMITY COUPLED TO A SUPERCONDUCTOR}

In the second model, we consider a one-band Rashba nanowire proximity coupled to an $s$-wave superconductor. The system is periodically driven by a time-dependent uniform magnetic field $B(t)$ of amplitude $B_{0}$ and frequency $\omega$ applied perpendicular to the SOI vector. We note that in the first model, the chemical potential was assumed to be close to the SOI energy. However, tuning of the chemical potential gets challenging if the system is coupled to a superconductor. Thus, our second model has an important advantage in that the chemical potential just needs to be below the SOI energy level [see Fig. 4] but does not need to be tuned to a particular value.
By adjusting $\omega$ of $B(t)$, one can then tune the Floquet Zeeman term to be resonant.

The Floquet driving takes place inside the same band. The lower (upper) energy states are labeled by the index $\eta=\overline{1}$ $(\eta=1)$ and spin up (down) by $\sigma=1(\sigma=\overline{1})$. The chemical potential $\mu_{\overline{1}}<0$ lies away from the SOI crossing. The frequency $\omega$ of $B(t)$ is chosen such that $\mu_{1}=\hbar \omega+\mu_{\overline{1}}$ satisfies the resonance condition both in energy and momentum space, see Fig. 4. The Fermi points in the two bands are given by $\left.k_{F \eta \sigma \pm}=\sigma k_{s o} \pm k_{s o} \sqrt{1+\left(\mu_{\eta} / E_{s o}\right)}\right]$. The driving frequency $\omega$ is determined by the condition $k_{F \overline{1} 1-}=k_{F 1 \overline{1}+}$. Again, to characterize the system, we linearize the Hamiltonian density around the Fermi points and keep only slowly varying fields [86]. The pairing term becomes

$$
H_{s}=\sum_{\eta} \Delta_{s c}\left[R_{\eta \overline{1}}^{\dagger} L_{\eta 1}^{\dagger}-R_{\eta 1}^{\dagger} L_{\eta \overline{1}}^{\dagger}+\text { H.c. }\right],
$$

where $\Delta_{s c}$ is the proximity induced superconducting gap. The resonant part of the Floquet term takes the form

$$
H_{d}=t_{F} \sum_{\eta}\left[R_{\eta \overline{1}}^{\dagger} L_{\bar{\eta} 1}+\text { H.c. }\right] .
$$

Here, $t_{F}=g \mu_{B} B_{0}$ is the amplitude of the Zeeman coupling in the Floquet representation.

The corresponding linearized Hamiltonian density belongs to the topological class DIII [85] is given by

$$
\mathcal{H}=\hbar v_{F} \hat{k} \tau_{3}+\Delta_{s c} \tau_{1} \sigma_{2} \delta_{2}+\frac{t_{F}}{2} \eta_{1} \delta_{3}\left(\tau_{1} \sigma_{1}+\tau_{2} \sigma_{2}\right),
$$

where $\delta_{i}$ are the Pauli matrices acting in electron-hole space. The spectrum reads $E_{1, \pm}= \pm \sqrt{\left(\hbar v_{F} k\right)^{2}+\Delta_{s c}^{2}}$ and $E_{2, \pm}^{2}=$ $\sqrt{\left(\hbar v_{F} k\right)^{2}+\left(t_{F} \pm \Delta_{s c}\right)^{2}}$, where $E_{1, \pm}$ is four- and $E_{2, \pm}$ twofold degenerate.

If the Floquet process dominates, $0<\Delta_{s c}<t_{F}$, the system is in the topological phase and hosts two zero-energy Majorana bound states at each of its ends protected by the effective timereversal symmetry, see Appendix B for details. In the presence of strong electron-electron interactions, we repeat the same bosonization procedure as described above, see Appendix C for details. We find that this setup can also be brought into the fractional topological regime and the many-body ground state consists of $\mathbb{Z}_{3}$ parafermions.

\section{CONCLUSIONS}

We proposed two simple one-dimensional setups which host zero-energy modes. In the first setup, we consider a single Rashba nanowire with applied uniform static magnetic field driven by a time-dependent electric field. An important feature of this scheme is that no superconductivity is needed, and thus no restrictions on the magnetic field strengths are required. Due to their intrinsic particle-hole symmetry, promising candidates for this setup are carbon nanotubes [68-71], graphene [72-76], and other two-dimensional crystals [77-83]. For example, the parameter estimates for metallic armchair graphene nanoribbons [75] are $\left(k_{B} T, t_{F}, \Delta_{Z}^{*}, E_{s o}\right)=(10,20,50,100) \mu \mathrm{eV}$, which correspond to $B=0.5 \mathrm{~T}$ (applied say, along the ribbon axis), $\omega=50 \mathrm{GHz}$ for $\mathcal{E} \approx 40 \mathrm{mV} / \mu \mathrm{m}\left(d_{c v} \approx 1 \mathrm{~nm}\right)$ applied transverse and in-plane. We note that the SOI can be 
generated by spatially rotating magnetic fields [75] or by using functionalized graphene [76].

In the second setup, we consider a model relying on superconductivity with the resonant driving achieved by applying a time-dependent magnetic field. The advantage of this one-band setup is the flexibility in the positioning of the chemical potential. This feature is especially valuable for semiconducting nanowires with large $g$ factor and with weak proximity-induced superconductivity [29,34]. The periodic driving brings both systems from the trivial to the topological phase. The systems can be tuned further from standard to fractional topological phase if strong electron-electron interactions are present, which leads in particular to the emergence of parafermions. The potential realization of such systems could be also in cold atoms or optical lattices. Relaxation and heating effects $[93,94]$ are of general concern in Floquet systems $[95,96]$. It has been shown, however, that these harmful effects can be suppressed by adiabatic buildup of the fractional state [45] or by engineered baths [97].

\section{ACKNOWLEDGMENTS}

We would like to acknowledge Peter Stano for useful discussions. This work was supported by the Swiss National Science Foundation (SNSF) and NCCR QSIT.

\section{APPENDIX A: ZERO-ENERGY BOUND STATE WAVE FUNCTIONS IN FLOQUET RASHBA NANOWIRE WITH APPLIED MAGNETIC FIELD}

Here we give the explicit wave function of the zero-energy bound state of the Hamiltonian given by Eq. (6) in the main text. As shown there, if $\Delta_{Z}>t_{F}>0$, the system belonging to the topological class DIII [85] is in the topological phase and hosts one localized zero-energy state at each wire end. The corresponding wave function of the state localized at the left end $(x=0)$ is given in the basis $\left(\Psi_{11}, \Psi_{1 \overline{1}}, \Psi_{\overline{1} 1}, \Psi_{\overline{1} \overline{1}}\right)$ by

$$
\begin{gathered}
\Phi(x)=\left(f(x), i f^{*}(x),-i f(x),-f^{*}(x)\right)^{T}, \\
f(x)=e^{-x / \xi_{t}} e^{-2 i k_{s o} x}-e^{-x / \xi_{-}},
\end{gathered}
$$

with the localization lengths defined as $\xi_{t}=\hbar v_{F} / t_{F}$ and $\xi_{-}=\hbar v_{F} /\left(\Delta_{Z}-t_{F}\right)$. This state is a fractional fermion of Jackiw-Rebbi type [98], and the topological band of our model can be mapped in particular to the Su-Schrieffer-Heeger (SSH) model $[91,99]$. Imposing the additional constraint that the system is half-filled (conservation of particle number [100]), such that only either the symmetric or the antisymmetric state, which is formed by the left- and right-end fermion state, is occupied, these fermions possess non-Abelian braid statistics [91] and can be used for quantum computing schemes, similar to Majorana zero modes. We note that this constraint reduces the degeneracy of the many-body ground state from four to two.

We further note that the particle-hole operator $U_{C}=\sigma_{2} \eta_{2}$ commutes with the Hamiltonian given in Eq. (6) of the main text. Thus, since the zero-energy bound state is nondegenerate, it follows that $\Phi(x)=U_{c} \Phi^{*}(x)$ (again, in complete analogy to the SSH model [99]). Similar to the SSH model, our system is topologically stable in the sense that the degeneracy of the many-body ground state cannot be lifted by local perturbations that respect the particle-hole symmetry. As was pointed out in Ref. [101], the SSH model (as well as our model) due to the intrinsic particle-hole symmetry resembles the Bogoliubov-de Gennes Hamiltonian of a one-dimensional topological superconductor hosting a nondegenerate single Majorana fermion at each end of the nanowire.

\section{APPENDIX B: MAJORANA FERMION WAVE FUNCTIONS IN FLOQUET RASHBA NANOWIRE WITH SUPERCONDUCTIVITY}

The Rashba nanowire driven by time-dependent magnetic field hosts two Majorana modes if $0<\Delta_{s c}<t_{F}$. The corresponding wave functions at the left wire end at $x=0$ are given in the basis composed of $\Psi_{\eta \sigma}$ $\left[\left(\Psi_{11}, \Psi_{1 \overline{1}}, \Psi_{11}^{\dagger}, \Psi_{1 \overline{1}}^{\dagger}, \Psi_{\overline{1} 1}, \Psi_{\overline{1} \overline{1}}, \Psi_{\overline{1} 1}^{\dagger}, \Psi_{\overline{1} \overline{1}}^{\dagger}\right)\right]$ by

$$
\begin{gathered}
\Phi_{M F 1}=\left(f, i f^{*}, f^{*},-i f, g, i g^{*}, g^{*},-i g\right)^{T}, \\
\Phi_{M F 2}=\left(-i f,-f^{*}, i f^{*},-f,-i g,-g^{*}, i g^{*},-g\right)^{T},
\end{gathered}
$$

$$
\begin{aligned}
& f=e^{-x / \xi_{-}} e^{i k_{F 1}-x}-e^{-x / \xi_{0}} e^{-i k_{F \overline{1} 1+} x}, \\
& g=e^{-x / \xi_{-}} e^{i k_{F 11-} x}-e^{-x / \xi_{0}} e^{-i k_{F 11+} x} .
\end{aligned}
$$

Here the localization lengths are given by $\xi_{0}=\hbar v_{F} / \Delta_{s c}$ and $\xi_{-}=\hbar v_{F} /\left(t_{F}-\Delta_{s c}\right)$. We note that the two Majorana fermion wave functions are connected by an effective time-reversal symmetry transformation, defined as the product of time reversal and band inversion symmetry transformations and given by $U_{T}=\sigma_{2} \tau_{1} \eta_{3}$. Under this symmetry transformation $U_{T}$ we find $R_{\eta \sigma} \rightarrow(\eta \sigma) L_{\eta \bar{\sigma}}$ and $L_{\eta \sigma} \rightarrow(\eta \sigma) R_{\eta \bar{\sigma}}$, and thus $U_{T}^{\dagger} \mathcal{H}^{*}(-k) U_{T}=\mathcal{H}(k)$. Again, the system is in the topological class DIII [85] and hosts one Kramers pair at each wire end in the topological phase unless the symmetries are broken. We note that, in contrast to Kramers pairs protected by the time-reversal symmetry [102-114], the degeneracy of the pair could potentially be lifted by a special type of disorder [115,116]. Importantly, however, we note that fluctuations in the chemical potential of the type $H_{d}=\sum_{\eta \sigma} \mu_{d} \Psi_{\eta \sigma}^{\dagger} \Psi_{\eta \sigma}$ affect both subbands in the same way and $H_{d}$ commutes with $U_{T}$. Thus, such fluctuations do not lift the degeneracy of the Kramers pair. The terms that break the $U_{T}$ symmetry but are not arising due to magnetic fields will be connected to the coupling between the two subbands. A term that could potentially break the degeneracy is given by $H_{d}=\sigma_{2} \tau_{1} \eta_{1}$. Such terms could arise from ac electric fields (which preserve time reversal invariance) combined with spin orbit interaction, giving rise to electrically driven spin resonance (EDSR). Thus, to minimize such instability effects care should be taken to have only $B$-field components over the wire size when driving the system.

\section{APPENDIX C: PARAFERMIONS IN FLOQUET RASHBA NANOWIRE WITH SUPERCONDUCTIVITY}

Similar to the first model considered in the main text, the periodically driven one-dimensional Rashba nanowire 


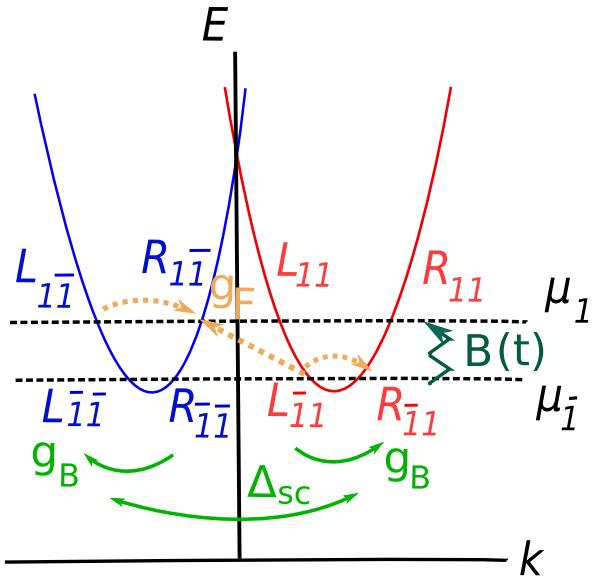

FIG. 5. The spectrum of the Rashba nanowire modified by the proximity gap $\Delta_{s c}$ and time-dependent magnetic field $B(t)$ in the strong electron-electron interaction regime. The index $\eta=1(\eta=\overline{1})$ is for upper (lower) band, $\sigma=1(\sigma=\overline{1})$ for spin up (red) [down (blue)]. The leading term in driving $H_{F}^{e e}$ (yellow arrows) involves two momentum-conserving backscattering terms. The superconductivity term $H_{s c}^{e e}$ (green arrows) commutes with $H_{F}^{e e}$ (orange arrows), therefore they can lead to simultaneous ordering of the corresponding bosonic fields, resulting in the fully gapped energy spectrum with zero-energy parafermion bound states localized at each wire end.

proximity coupled to a superconductor can also be brought into the fractional topological regime. The frequency of the ac magnetic field is chosen to be $\hbar \omega=\mu_{1}-\mu_{\overline{1}}$, where the chemical potentials are fixed such that $\left(k_{F \overline{1} \overline{1}+}-k_{F \overline{1} \overline{1}_{-}}\right)+$ $\left(k_{F 11+}-k_{F 11-}\right)=\left(k_{F \overline{1} 1+}-k_{F 11-}\right) \quad$ or $\quad\left(k_{F \overline{1} 1+}-k_{F \overline{1} 1-}\right)+$ $\left(k_{F 1 \overline{1}+}-k_{F 1 \overline{1}-}\right)=\left(k_{F 1 \overline{1}+}-k_{F \overline{1} 1-}\right)$ [see Fig. 5(b)]. Again, we assume that the driving term $H_{F}^{e e}$ describes the dominant process.

Hence, the leading order term that conserves momentum is given by

$$
\begin{aligned}
H_{F}^{e e}= & g_{F}\left[\left(R_{1 \overline{1}}^{\dagger} L_{\overline{1} 1}\right)\left(R_{\overline{1} 1}^{\dagger} L_{\overline{1} 1}\right)\left(R_{1 \overline{1}}^{\dagger} L_{1 \overline{1}}\right)\right. \\
& \left.+\left(R_{\overline{1} \overline{1}}^{\dagger} L_{11}\right)\left(R_{\overline{1} \overline{1}}^{\dagger} L_{\overline{1} \overline{1}}\right)\left(R_{11}^{\dagger} L_{11}\right)+\text { H.c. }\right] .
\end{aligned}
$$

The superconducting term which commutes with $H_{F}^{e e}$, is given by

$$
\begin{aligned}
H_{s c}^{e e}= & g_{s c}\left[\left(R_{11}^{\dagger} L_{1 \overline{1}}^{\dagger}\right)\left(R_{11}^{\dagger} L_{11}\right)\left(R_{1 \overline{1}} L_{1 \overline{1}}^{\dagger}\right)\right. \\
& \left.+\left(R_{\overline{1} 1}^{\dagger} L_{\overline{1} \overline{1}}^{\dagger}\right)\left(R_{\overline{1} 1}^{\dagger} L_{\overline{1} 1}\right)\left(R_{\overline{1} \overline{1}} L_{\overline{1} \overline{1}}^{\dagger}\right)+\text { H.c. }\right],
\end{aligned}
$$

where $g_{F} \propto t_{F} g_{B}^{2}$ and $g_{s c} \propto \Delta_{s c} g_{B}^{2}$. We note that these terms are possible only due to backscattering events of finite strength $g_{B}$. We use bosonic fields $\phi_{r \eta \sigma}$ as $R_{\eta \sigma}=e^{i \phi_{1 \eta \sigma}}$ and $L_{\eta \sigma}=e^{i \phi_{\overline{1} \eta \sigma}}$ with the only nonzero commutation relations given by $\left[\phi_{r \eta \sigma}(x), \phi_{r^{\prime} \eta^{\prime} \sigma^{\prime}}\left(x^{\prime}\right)\right]=i \pi r \delta_{r r^{\prime}} \delta_{\eta \eta^{\prime}} \delta_{\sigma \sigma^{\prime}} \operatorname{sgn}(x-$ $\left.x^{\prime}\right)$. The problem simplifies by using new fields, therefore introducing $\tilde{\phi}_{r \eta \sigma}=\left(2 \phi_{r \eta \sigma}-\phi_{\bar{r} \eta \sigma}\right) / 3$ with $\left[\tilde{\phi}_{r \eta \sigma}(x), \tilde{\phi}_{r^{\prime} \eta^{\prime} \sigma^{\prime}}\left(x^{\prime}\right)\right]=$ $\operatorname{ir}(\pi / 3) \delta_{r r^{\prime}} \delta_{\eta \eta^{\prime}} \delta_{\sigma \sigma^{\prime}} \operatorname{sgn}\left(x-x^{\prime}\right)$. In terms of the new fields, the nonquadratic Hamiltonian takes the form

$$
\begin{aligned}
& H_{s c}^{e e}=2 g_{s c} \sum_{\eta} \cos \left[3\left(\tilde{\phi}_{1 \eta 1}+\tilde{\phi}_{\overline{1} \eta \overline{1}}\right)\right], \\
& H_{F}^{e e}=2 g_{F} \sum_{\eta} \cos \left[3\left(\tilde{\phi}_{1 \eta \overline{1}}-\tilde{\phi}_{\overline{1} \bar{\eta} 1}\right)\right] .
\end{aligned}
$$

Again, we double the system size and halve the number of fields in order to satisfy vanishing boundary conditions at the two ends of the system $[42,43]$. The new fields can be written as

$$
\begin{aligned}
& \chi_{1 \eta}(x)= \begin{cases}\tilde{\phi}_{1 \eta 1}(x), & x>0 \\
\tilde{\phi}_{\overline{1} \eta 1}(-x)+\pi, & x<0\end{cases} \\
& \chi_{\overline{1} \eta}(x)= \begin{cases}\tilde{\phi}_{\overline{1} \eta \overline{1}}(x), & x>0 \\
\tilde{\phi}_{1 \eta \overline{1}}(-x)+\pi, & x<0\end{cases}
\end{aligned}
$$

Therefore, the Hamiltonian has the following form

$$
H^{e e}=\left\{\begin{array}{ll}
2 g_{s c} \sum_{\eta} \cos \left[3\left(\chi_{1 \eta}+\chi_{\overline{1} \eta}\right)\right], & x>0 \\
2 g_{F} \sum_{\eta} \cos \left[3\left(\chi_{1 \bar{\eta}}-\chi_{\overline{1} \eta}\right)\right], & x<0
\end{array}\right. \text {. }
$$

Next, we transform the chiral fields to conjugate fields $\phi$ 's and $\theta$ 's as $\chi_{r \eta}=\left[r \phi_{2}+\theta_{2}+\eta\left(r \phi_{1}+\theta_{1}\right) / 3\right] / 2$ and get

$$
H^{e e}=\left\{\begin{array}{ll}
4 g_{s c} \cos \left(\theta_{1}\right) \cos \left(3 \theta_{2}\right), & x>0 \\
4 g_{F} \cos \left(\theta_{1}\right) \cos \left(3 \phi_{2}\right), & x<0
\end{array} .\right.
$$

To minimize the energy of the system $[42,43]$, we find $\theta_{1}=$ $\pi \hat{M}$ (pinned uniformly over the entire wire), $\phi_{2}=\pi(1+\hat{M}+$ $2 \hat{n}) / 3$ for $x<0$, and $\theta_{2}=\pi(1+\hat{M}+2 \hat{l}) / 3$ for $x>0$. Thus, a domain wall is formed between two noncommuting fields, namely $\phi_{2}$ and $\theta_{2},\left[\phi_{2}(x), \theta_{2}\left(x^{\prime}\right)\right]=-i \pi / 3 \operatorname{sgn}\left(x-x^{\prime}\right)$. This gives the nonzero commutator $[\hat{n}, \hat{l}]=3 i / 4 \pi$, hence we define two operators which commute with the Hamiltonian and are at zero energy [42,43],

$$
\alpha_{1}=e^{i 4 \pi(\hat{l}-\hat{n}) / 3} ; \alpha_{\overline{1}}=e^{i 4 \pi(\hat{l}+\hat{n}) / 3} .
$$

These zero energy operators satisfy the parafermionic algebra: $\alpha_{1}^{3}=\alpha_{\overline{1}}^{3}=1$ and $\alpha_{1} \alpha_{\overline{1}}=e^{-2 i \pi / 3} \alpha_{\overline{1}} \alpha_{1}$.
[1] M. Z. Hasan and C. L. Kane, Rev. Mod. Phys. 82, 3045 (2010).

[2] X.-L. Qi and S.-C. Zhang, Rev. Mod. Phys. 83, 1057 (2011).

[3] G. Tkachov and E. M. Hankiewicz, Phys. Status Solidi B 250, 215 (2013).

[4] B. A. Volkov and O. A. Pankratov, Pis'ma Zh. Eksp. Teor. Fiz. 42, 145 (1985) [JETP Lett. 42, 178 (1985)].

[5] O. A. Pankratov, S. V. Pakhomov, and B. A. Volkov, Solid State Commun. 61, 93 (1987).
[6] B. A. Bernevig, T. L. Hughes, and S.-C. Zhang, Science 314, 1757 (2006)

[7] M. König, S. Wiedmann, C. Brune, A. Roth, H. Buhmann, L. W. Molenkamp, X.-L. Qi, and S.-C. Zhang, Science 318, 766 (2007).

[8] A. Roth, C. Brune, H. Buhmann, L. W. Molenkamp, J. Maciejko, X.-L. Qi, and S.-C. Zhang, Science 325, 294 (2009).

[9] Y. Tanaka, T. Yokoyama, and N. Nagaosa, Phys. Rev. Lett. 103, 107002 (2009). 
[10] K. C. Nowack, E. M. Spanton, M. Baenninger, M. König, J. R. Kirtley, B. Kalisky, C. Ames, P. Leubner, C. Brune, H. Buhmann, L. W. Molenkamp, D. Goldhaber-Gordon, and K. A. Moler, Nat. Mater. 12, 787 (2013).

[11] S. Hart, H. Ren, T. Wagner, P. Leubner, M. Mühlbauer, C. Brüne, H. Buhmann, L. W. Molenkamp, and A. Yacoby, Nat. Phys. 10, 638 (2014).

[12] V. S. Pribiag, A. J. A. Beukman, F. Qu, M. C. Cassidy, C. Charpentier, W. Wegscheider, and L. P. Kouwenhoven, Nat. Nanotechnol. 10, 593 (2015).

[13] E. Sagi and Y. Oreg, Phys. Rev. B 90, 201102 (2014).

[14] M. Sato and S. Fujimoto, Phys. Rev. B 79, 094504 (2009).

[15] R. M. Lutchyn, J. D. Sau, and S. Das Sarma, Phys. Rev. Lett. 105, 077001 (2010).

[16] Y. Oreg, G. Refael, and F. von Oppen, Phys. Rev. Lett. 105, 177002 (2010).

[17] J. Alicea, Phys. Rev. B 81, 125318 (2010).

[18] S. Gangadharaiah, B. Braunecker, P. Simon, and D. Loss, Phys. Rev. Lett. 107, 036801 (2011).

[19] A. C. Potter and P. A. Lee, Phys. Rev. B 83, 094525 (2011).

[20] J. Klinovaja, S. Gangadharaiah, and D. Loss, Phys. Rev. Lett. 108, 196804 (2012).

[21] D. Chevallier, D. Sticlet, P. Simon, and C. Bena, Phys. Rev. B 85, 235307 (2012).

[22] J. Klinovaja, G. J. Ferreira, and D. Loss, Phys. Rev. B 86, 235416 (2012).

[23] D. Sticlet, C. Bena, and P. Simon, Phys. Rev. Lett. 108, 096802 (2012).

[24] J. Klinovaja, P. Stano, and D. Loss, Phys. Rev. Lett. 109, 236801 (2012).

[25] S. Nadj-Perge, I. K. Drozdov, B. A. Bernevig, and A. Yazdani, Phys. Rev. B 88, 020407(R) (2013).

[26] J. Klinovaja, P. Stano, A. Yazdani, and D. Loss, Phys. Rev. Lett. 111, 186805 (2013).

[27] B. Braunecker and P. Simon, Phys. Rev. Lett. 111, 147202 (2013).

[28] M. M. Vazifeh and M. Franz, Phys. Rev. Lett. 111, 206802 (2013).

[29] V. Mourik, K. Zuo, S. M. Frolov, S. R. Plissard, E. P. A. M. Bakkers, and L. P. Kouwenhoven, Science 336, 1003 (2012).

[30] M. T. Deng, C. L. Yu, G. Y. Huang, M. Larsson, P. Caroff, and H. Q. Xu, Nano Lett. 12, 6414 (2012).

[31] A. Das, Y. Ronen, Y. Most, Y. Oreg, M. Heiblum, and H. Shtrikman, Nat. Phys. 8, 887 (2012).

[32] L. P. Rokhinson, X. Liu, and J. K. Furdyna, Nat. Phys. 8, 795 (2012).

[33] J. R. Williams, A. J. Bestwick, P. Gallagher, S. S. Hong, Y. Cui, A. S. Bleich, J. G. Analytis, I. R. Fisher, and D. GoldhaberGordon, Phys. Rev. Lett. 109, 056803 (2012).

[34] H. O. H. Churchill, V. Fatemi, K. Grove-Rasmussen, M. T. Deng, P. Caroff, H. Q. Xu, and C. M. Marcus, Phys. Rev. B 87, 241401(R) (2013).

[35] S. Nadj-Perge, I. K. Drozdov, J. Li, H. Chen, S. Jeon, J. Seo, A. H. MacDonald, B. A. Bernevig, and A. Yazdani, Science 346, 602 (2014).

[36] R. Pawlak, M. Kisiel, J. Klinovaja, T. Meier, S. Kawai, T. Glatzel, D. Loss, and E. Meyer, npj Quantum Information 2, 16035 (2016).

[37] M. Barkeshli, C.-M. Jian, and X.-L. Qi, Phys. Rev. B 87, 045130 (2013).
[38] N. H. Lindner, E. Berg, G. Refael, and A. Stern, Phys. Rev. X 2, 041002 (2012).

[39] D. J. Clarke, J. Alicea, and K. Shtengel, Nat. Commun. 4, 1348 (2013).

[40] M. Cheng, Phys. Rev. B 86, 195126 (2012).

[41] R. S. K. Mong, D. J. Clarke, J. Alicea, N. H. Lindner, P. Fendley, C. Nayak, Y. Oreg, A. Stern, E. Berg, K. Shtengel, and M. P. A. Fisher, Phys. Rev. X 4, 011036 (2014).

[42] J. Klinovaja and D. Loss, Phys. Rev. Lett. 112, 246403 (2014).

[43] Y. Oreg, E. Sela, and A. Stern, Phys. Rev. B 89, 115402 (2014).

[44] A. Vaezi, Phys. Rev. X 4, 031009 (2014).

[45] N. H. Lindner, G. Refael, and V. Galitski, Nat. Phys. 7, 490 (2011).

[46] T. Kitagawa, M. A. Broome, A. Fedrizzi, M. S. Rudner, E. Berg, I. Kassal, A. Aspuru-Guzik, E. Demler, and A. G. White, Nat. Commun. 3, 882 (2012).

[47] M. C. Rechtsman, J. M. Zeuner, Y. Plotnik, Y. Lumer, D. Podolsky, F. Dreisow, S. Nolte, M. Segev, and A. Szameit, Nature (London) 496, 196 (2013).

[48] J. Klinovaja, P. Stano, and D. Loss, Phys. Rev. Lett. 116, 176401 (2016).

[49] Y. T. Katan and D. Podolsky, Phys. Rev. Lett. 110, 016802 (2013).

[50] K. Plekhanov, G. Roux, and K. Le Hur, Phys. Rev. B 95, 045102 (2017).

[51] M. S. Foster, V. Gurarie, M. Dzero, and E. A. Yuzbashyan, Phys. Rev. Lett. 113, 076403 (2014).

[52] X. Zhang, T. Ong, and N. Nagaosa, Phys. Rev. B 94, 235137 (2016).

[53] T. Kitagawa, E. Berg, M. Rudner, and E. Demler, Phys. Rev. B 82, 235114 (2010).

[54] N. H. Lindner, D. L. Bergman, G. Refael, and V. Galitski, Phys. Rev. B 87, 235131 (2013).

[55] J.-I. Inoue and A. Tanaka, Phys. Rev. Lett. 105, 017401 (2010).

[56] M. S. Rudner, N. H. Lindner, E. Berg, and M. Levin, Phys. Rev. X 3, 031005 (2013).

[57] M. Thakurathi, K. Sengupta, and D. Sen, Phys. Rev. B 89, 235434 (2014).

[58] D. E. Liu, A. Levchenko, and H. U. Baranger, Phys. Rev. Lett. 111, 047002 (2013).

[59] A. A. Reynoso and D. Frustaglia, Phys. Rev. B 87, 115420 (2013).

[60] P. Delplace, A. Gomez-Leon, and G. Platero, Phys. Rev. B 88, 245422 (2013).

[61] A. G. Grushin, A. Gomez-Leon, and T. Neupert, Phys. Rev. Lett. 112, 156801 (2014).

[62] M. Thakurathi, A. A. Patel, D. Sen, and A. Dutta, Phys. Rev. B 88, 155133 (2013).

[63] V. Dal Lago, M. Atala, and L. E. F. Foa Torres, Phys. Rev. A 92, 023624 (2015).

[64] T. Oka and H. Aoki, Phys. Rev. B 79, 081406 (2009).

[65] S. Roy and G. J. Sreejith, Phys. Rev. B 94, 214203 (2016).

[66] M. Bukov, M. Kolodrubetz, and A. Polkovnikov, Phys. Rev. Lett. 116, 125301 (2016).

[67] A. Agarwala and D. Sen, Phys. Rev. B 95, 014305 (2017).

[68] F. Kuemmeth, S. Ilani, D. C. Ralph, and P. L. McEuen, Nature (London) 452, 448 (2008).

[69] G. A. Steele, G. Gotz, and L. P. Kouwenhoven, Nat. Nanotechnol. 4, 363 (2009). 
[70] W. Izumida, K. Sato, and R. Saito, J. Phys. Soc. Jpn. 78, 074707 (2009).

[71] J. Klinovaja, M. J. Schmidt, B. Braunecker, and D. Loss, Phys. Rev. B 84, 085452 (2011).

[72] K. S. Novoselov, A. K. Geim, S. V. Morozov, D. Jiang, M. I. Katsnelson, I. V. Grigorieva, S. V. Dubonos, and A. A. Firsov, Nature (London) 438, 197 (2005).

[73] M. Y. Han, B. Ozyilmaz, Y. Zhang, and P. Kim, Phys. Rev. Lett. 98, 206805 (2007).

[74] D. Marchenko, A. Varykhalov, M. R. Scholz, G. Bihlmayer, E. I. Rashba, A. Rybkin, A. M. Shikin, and O. Rader, Nat. Commun. 3, 1232 (2012).

[75] J. Klinovaja and D. Loss, Phys. Rev. X 3, 011008 (2013).

[76] Z. Wang, D. K. Ki, H. Chen, H. Berger, A. H. MacDonald, and A. F. Morpurgo, Nat. Commun. 6, 8339 (2015).

[77] D. Costanzo, S. Jo, H. Berger, and A. F. Morpurgo, Nat. Nanotechnol. 11, 339 (2016).

[78] M. Remskar, A. Mrzel, Z. Skraba, A. Jesih, M. Ceh, J. Demšar, P. Stadelmann, F. Lévy, and D. Mihailovic, Science 292, 479 (2001).

[79] B. Radisavljevic, A. Radenovic, J. Brivio, V. Giacometti, and A. Kis, Nat. Nanotechnol. 6, 147 (2011).

[80] Q. H. Wang, K. Kalantar-Zadeh, A. Kis, J. N. Coleman, and M. S. Strano, Nat. Nanotechnol. 7, 699 (2012).

[81] Y. Huang et al., Nano Research 6, 200 (2013).

[82] A. Kormanyos, V. Zolyomi, N. D. Drummond, and G. Burkard, Phys. Rev. X 4, 011034 (2014).

[83] A. Kormanyos, G. Burkard, M. Gmitra, J. Fabian, V. Zlyomi, N. D. Drummond, and V. I. Fal'ko, 2D Materials 2, 022001 (2015).

[84] J. H. Shirley, Phys. Rev. 138, B979 (1965).

[85] S. Ryu, A. P. Schnyder, A. Furusaki, and A. W. W. Ludwig, New J. Phys. 12, 065010 (2010).

[86] J. Klinovaja and D. Loss, Phys. Rev. B 86, 085408 (2012).

[87] J. Klinovaja and D. Loss, Eur. Phys. J. B 88, 62 (2015).

[88] L. Fu, Phys. Rev. Lett. 106, 106802 (2011).

[89] Y. Ando and L. Fu, Annu. Rev. Condens. Matter Phys. 6, 361 (2015).

[90] A. A. Burkov and L. Balents, Phys. Rev. Lett. 107, 127205 (2011).

[91] J. Klinovaja and D. Loss, Phys. Rev. Lett. 110, 126402 (2013).
[92] A. H. Castro Neto, F. Guinea, N. M. R. Peres, K. S. Novoselov, and A. K. Geim, Rev. Mod. Phys. 81, 109 (2009).

[93] G. M. Eliashberg, JETP Lett. 11, 114 (1970).

[94] L. I. Glazman, Sov. Phys. JETP 53, 178 (1981).

[95] H. Dehghani, T. Oka, and A. Mitra, Phys. Rev. B 90, 195429 (2014).

[96] T. Iadecola, T. Neupert, and C. Chamon, Phys. Rev. B 91, 235133 (2015).

[97] K. I. Seetharam, C.-E. Bardyn, N. H. Lindner, M. S. Rudner, and G. Refael, Phys. Rev. X 5, 041050 (2015).

[98] R. Jackiw and C. Rebbi, Phys. Rev. D 13, 3398 (1976).

[99] W. P. Su, J. R. Schrieffer, and A. J. Heeger, Phys. Rev. Lett. 42, 1698 (1979).

[100] M. Barkeshli and X. L. Qi, Phys. Rev. X 2, 031013 (2012).

[101] C. Kane, Topological Insulators, edited by M. Franz and L. Molenkamp (Elsevier, Vancouver, 2013), Chap. I.

[102] C. L. M. Wong and K. T. Law, Phys. Rev. B 86, 184516 (2012).

[103] S. Nakosai, J. C. Budich, Y. Tanaka, B. Trauzettel, and N. Nagaosa, Phys. Rev. Lett. 110, 117002 (2013).

[104] X.-J. Liu, C. L. M. Wong, and K. T. Law, Phys. Rev. X 4, 021018 (2014).

[105] E. Dumitrescu, J. D. Sau, and S. Tewari, Phys. Rev. B 90, 245438 (2014).

[106] F. Zhang, C. L. Kane, and E. J. Mele, Phys. Rev. Lett. 111, 056402 (2013).

[107] A. Keselman, L. Fu, A. Stern, and E. Berg, Phys. Rev. Lett. 111, 116402 (2013).

[108] A. Haim, A. Keselman, E. Berg, and Y. Oreg, Phys. Rev. B 89, 220504 (2014).

[109] J. Klinovaja and D. Loss, Phys. Rev. B 90, 045118 (2014).

[110] E. Gaidamauskas, J. Paaske, and K. Flensberg, Phys. Rev. Lett. 112, 126402 (2014).

[111] J. Klinovaja, A. Yacoby, and D. Loss, Phys. Rev. B 90, 155447 (2014).

[112] C.-X. Liu and B. Trauzettel, Phys. Rev. B 83, 220510(R) (2011).

[113] C. Schrade, A. A. Zyuzin, J. Klinovaja, and D. Loss, Phys. Rev. Lett. 115, 237001 (2015).

[114] E. A. Mellars and B. Beri, Phys. Rev. B 94, 174508 (2016).

[115] S. Hoffman, J. Klinovaja, and D. Loss, Phys. Rev. B 93, 165418 (2016).

[116] C.-H. Hsu, P. Stano, J. Klinovaja, and D. Loss, Phys. Rev B 92, 235435 (2015). 